\title{
An Analysis of the Flow of a Newtonian Fluid between Two Moving Parallel Plates
}

\author{
A. Zeb, ${ }^{1}$ A. M. Siddiqui, ${ }^{2}$ and M. Ahmed ${ }^{3}$ \\ ${ }^{1}$ Department of Mathematics and Statistics, Al Imam Mohammad Ibn Saud Islamic University, \\ P.O. Box 90950, Riyadh 11623, Saudi Arabia \\ ${ }^{2}$ Department of Mathematics, Pennsylvania State University, York Campus, Edgecomb Avenue, York, PA 17403, USA \\ ${ }^{3}$ Department of Mathematics, COMSATS Institute of Information Technology, Park Road, \\ Chak Shahzad, Islamabad 44000, Pakistan \\ Correspondence should be addressed to A.Zeb; amtaz56@yahoo.co.uk
}

Received 16 July 2013; Accepted 2 September 2013

Academic Editors: K. Lurie and M. Musso

Copyright (C) 2013 A. Zeb et al. This is an open access article distributed under the Creative Commons Attribution License, which permits unrestricted use, distribution, and reproduction in any medium, provided the original work is properly cited.

We consider flow of an incompressible Newtonian fluid produced by two parallel plates, moving towards and away from each other with constant velocity. Inverse solutions of the equations of motion are obtained by assuming certain forms of the stream function. Analytical expressions for the stream function, fluid velocity components, and fluid pressure are derived.

\section{Introduction}

Owing to the nonlinear nature of the Navier-Stokes equations, their exact solutions are far and few in number. Importance of the exact solutions lies in the fact that they serve as standards for validating the corresponding solutions obtained by numerical methods and other approximate techniques. The inverse or the indirect method, see for example, Neményi [1], is often used to compute these exact solutions.

Finding exact solution using the inverse method consists of making an assumption on the general form of the stream function $\psi$, involving certain unknown functions, without considering the shape of boundaries of the solution domain occupied by the fluid. We then substitute this assumed form of $\psi$ in the compatibility equation for the stream function to find the unknown functions involved in $\psi$. This provides the stream function $\psi$, and subsequently, the fluid velocity components. Once the fluid velocity components are available, then the second step is to compute the fluid pressure field using the component form of the Navier-Stokes equations. This kind of methods with applications in various fields of continuum mechanics are given in an article by Neményi [1]. Moreover, a number of reviews on the exact solutions for Navier-Stokes equations have been published, for example, Berker [2], Dryden et al. [3], Whitham [4], Schlichting [5], Wang [6], and Wang [7].

In this paper, we apply the technique described above to analyze the flow of a viscous incompressible fluid induced by the motion of two parallel plates. These plates are moving towards each other and in the opposite direction with a constant velocity $\alpha$, when size of the plates is much larger than distance between them. A large class of the processes such as the motion of liquid through a hydraulic pump and that of the underground water may mathematically be considered from this point of view, see Aristov and Gitman [8] and Siddiqui et al. $[9,10]$. We apply the inverse method to solve this problem for Berker type flow, Riabouchinsky type flow and the potential flow with perturbation. The results obtained are compared with the known viscous solutions by setting the relative velocity $\alpha$ of the disks equal to zero.

\section{Basic Equations}

If $\mathbf{u}$ denotes the fluid velocity, $\mathbf{f}$ the total of body forces, $\mathrm{T}$ the stress tensor, $\rho$ the constant fluid density, and $D / D t=(\partial / \partial t)+\mathbf{u} \cdot \nabla$ the material derivative, then the basic 
set of equations governing the flow of an incompressible fluid are

$$
\begin{gathered}
\nabla \cdot \mathbf{u}=0, \\
\rho \frac{D \mathbf{u}}{D t}=\rho \mathbf{f}+\nabla \cdot \mathbf{T} .
\end{gathered}
$$

The constitutive equation, relating the stress tensor to the rate of strain tensor, for an incompressible Newtonian fluid is, $\mathbf{T}=-p \mathbf{I}+\mu \nabla \mathbf{u}$. Thus, in the absence of body forces, the basic set of equations governing the flow of an incompressible fluid becomes

$$
\begin{gathered}
\nabla \cdot \mathbf{u}=0 \\
\frac{\partial \mathbf{u}}{\partial t}+(\mathbf{u} \cdot \nabla) \mathbf{u}=-\frac{1}{\rho} \nabla p+\nu \nabla^{2} \mathbf{u}
\end{gathered}
$$

where $p$ denotes the fluid pressure, $\mu$ the coefficient of viscosity, and $\nu=\mu / \rho$ the kinematic viscosity.

\section{Problem Formulation}

Consider the motion of an incompressible Newtonian fluid produced by the motion of two parallel plates moving towards each other or in the opposite direction with constant velocity $\alpha$. The size of the disks is much larger than the distance between them. We assume that the horizontal velocity components $u$ and $v$ are independent of vertical coordinate $z$, whereas the vertical velocity component $w$ depends linearly on the distance between the disks, see Aristov and Gitman [8] and Siddiqui et al. $[9,10]$.

$$
\mathbf{u}=[u(x, y, t), v(x, y, t),-2 \alpha z] .
$$

Using (3) into (2), we obtain

$$
\begin{gathered}
\frac{\partial u}{\partial x}+\frac{\partial v}{\partial y}=2 \alpha \\
\frac{\partial u}{\partial t}+u \frac{\partial u}{\partial x}+v \frac{\partial u}{\partial y}+\frac{\partial p^{*}}{\partial x}=\nu \nabla^{2} u \\
\frac{\partial v}{\partial t}+u \frac{\partial v}{\partial x}+v \frac{\partial v}{\partial y}+\frac{\partial p^{*}}{\partial y}=\nu \nabla^{2} v
\end{gathered}
$$

where $p^{*}=p(x, y, t) / \rho-2 \alpha^{2} z^{2}$. The equation for the vertical component $w$ is identically satisfied. Equations (4)(6) are three partial differential equations in three unknowns, namely, $u, v$, and $p^{*}$. Once the velocity components $u$ and $v$ are determined, the pressure field can be found through (5) and (6). Eliminating $p^{*}$ between (5) and (6), we obtain

$$
\frac{\partial \omega}{\partial t}+2 \alpha \omega+u \frac{\partial \omega}{\partial x}+v \frac{\partial \omega}{\partial y}=v \nabla^{2} \omega, \quad \omega=\frac{\partial v}{\partial x}-\frac{\partial u}{\partial y},
$$

where $\omega$ denotes magnitude of the vorticity vector.

We now introduce the stream function $\psi$ defining the horizontal components of the fluid velocity through the relations $[8,9]$ :

$$
u=\alpha x+\frac{\partial \psi}{\partial y}, \quad v=\alpha y-\frac{\partial \psi}{\partial x}
$$

Then, the continuity equation (4) is identically satisfied and momentum equations (5) and (6) yield the following compatibility equation:

$$
\begin{aligned}
& \frac{\partial\left(\nabla^{2} \psi\right)}{\partial t}-\frac{\partial\left(\psi, \nabla^{2} \psi\right)}{\partial(x, y)} \\
& \quad=-\alpha\left[2 \nabla^{2} \psi+x \frac{\partial\left(\nabla^{2} \psi\right)}{\partial x}+y \frac{\partial\left(\nabla^{2} \psi\right)}{\partial y}\right]+\nu \nabla^{4} \psi
\end{aligned}
$$

where

$$
\frac{\partial\left(\psi, \nabla^{2} \psi\right)}{\partial(x, y)}=\frac{\partial \psi}{\partial x} \frac{\partial\left(\nabla^{2} \psi\right)}{\partial y}-\frac{\partial \psi}{\partial y} \frac{\partial\left(\nabla^{2} \psi\right)}{\partial x} .
$$

The stream function $\psi$ is determined by solving the compatibility equation (9), and the velocity components $u$ and $v$ can be determined through (8). One solution of the compatibility equation (9) is the trivial solution $\psi=0$ that corresponds to the potential motion, known as the motion near stagnation point. Other stationary solutions are examined in the following section.

\section{Solutions of the Problem}

Berker Type Flows. In this type of flow we look for the stream function $\psi$ in the following general form

$$
\psi(x, y)=F(x)+G(y),
$$

where $F(x)$ and $G(y)$ are arbitrary functions of $x$ and $y$, respectively. This type of flow was also studied in the context of second grade fluid by Siddiqui et al. $[9,10]$ using certain forms of $F(x)$ and $G(y)$. Here, we consider more general cases. Using (11) in the compatibility equation (9), we obtain

$$
\begin{aligned}
F^{\prime \prime \prime}(x) G^{\prime}(y)-F^{\prime}(x) G^{\prime \prime \prime}(y) \\
=-\alpha\left[2 F^{\prime \prime}(x)+2 G^{\prime \prime}(y)+x F^{\prime \prime \prime}(x)+y G^{\prime \prime \prime}(y)\right] \\
\quad+v\left[F^{(4)}(x)+G^{(4)}(y)\right] .
\end{aligned}
$$

For $\alpha=0$ (12) reduces to Berker's case. Now following Berker [2], we plan to study the following particular cases.

If $F^{\prime \prime}(x)=0, G^{\prime \prime}(y) \neq 0$. Then, in this case the vorticity $\omega=-\nabla^{2} \psi$ is a function of $y$ only and (12) reduces to

$$
G^{(4)}(y)-\left(\frac{\alpha y-A_{1}}{v}\right) G^{\prime \prime \prime}(y)-\left(\frac{2 \alpha}{v}\right) G^{\prime \prime}(y)=0,
$$

where $F^{\prime}(x)=A_{1}, A_{1}$ being an arbitrary constant. Now, integrate (13) twice to get

$$
G^{\prime \prime}(y)-\left(\frac{\alpha y-A_{1}}{v}\right) G^{\prime}(y)=A y+B,
$$

where $A, B$ are arbitrary constants. For $A=B=0$, the solution of (14) is given by

$$
G(y)=\sigma_{1} \sum_{n=1}^{\infty}\left(\frac{\alpha^{n-1}\left(y-A_{1} / \alpha\right)^{2 n-1}}{(2 n-1)(n-1) !(2 \nu)^{n-1}}\right),
$$


where $\sigma_{1}=\exp \left[K_{1}-A_{1}^{2} /(2 \nu \alpha)\right]$ and $K_{1}$ denotes an arbitrary constant. Therefore, the stream function $\psi$ is given by

$$
\begin{aligned}
\psi(x, y)= & A_{1} x+B_{1} \\
& +\sigma_{1} \sum_{n=1}^{\infty}\left(\frac{\alpha^{n-1}\left(y-A_{1} / \alpha\right)^{2 n-1}}{(2 n-1)(n-1) !(2 \nu)^{n-1}}\right),
\end{aligned}
$$

where $B_{1}$ is an arbitrary constant. Although the solution (16) for the stream function $\psi$ is a power series, we were able to identify the series solution for $u$. Thus, the velocity components are

$$
\begin{gathered}
u=\alpha x+\sigma_{1} \exp \left(\frac{\alpha\left(y-A_{1} / \alpha\right)^{2}}{2 \nu}\right), \\
v=\alpha y-A_{1}, \\
w=-2 \alpha z .
\end{gathered}
$$

Consequently, the analytical expression for the fluid pressure distribution in this case is the following:

$$
\begin{aligned}
p= & a_{1} \eta x-\frac{\alpha^{2} \rho}{2} \\
& \times\left[x^{2}+\left(y-\frac{A_{1}}{\alpha}\right)^{2}+b_{1}\right]-2 \rho \alpha^{2} z^{2}, \quad b_{1}=\frac{2 c_{1}}{\alpha^{2} \rho},
\end{aligned}
$$

where $a_{1}$ and $c_{1}$ are arbitrary constants.

Similarly, if $F^{\prime \prime}(x) \neq 0, G^{\prime \prime}(y)=0$, then the stream function $\psi$ in the form of a power series is given by

$$
\begin{aligned}
\psi(x, y)= & \sigma_{2} \sum_{n=1}^{\infty}\left(\frac{\alpha^{n-1}\left(x+A_{2} \alpha\right)^{2 n-1}}{(2 n-1)(n-1) !(2 \nu)^{n-1}}\right) \\
& +A_{2} y+B_{2},
\end{aligned}
$$

where $\sigma_{2}=\exp \left[K_{2}-A_{2}^{2} /(2 v \alpha)\right], A_{2}=G^{\prime}(y)$. Here, $A_{2}, B_{2}$, and $K_{2}$ are arbitrary constants. Again, we were able to identify the power series for $v$, and components of the fluid velocity are

$$
\begin{gathered}
u=\alpha x+A_{2}, \\
v=\alpha y-\sigma_{2} \exp \left(\frac{\alpha\left(x+A_{2} / \alpha\right)^{2}}{2 v}\right), \\
w=-2 \alpha z .
\end{gathered}
$$

Correspondingly, the analytical expression for the fluid pressure distribution in this case is

$$
\begin{aligned}
p= & a_{2} \eta y-\frac{\alpha^{2} \rho}{2} \\
& \times\left[\left(x+\frac{A_{2}}{\alpha}\right)^{2}+y^{2}+b_{2}\right]-2 \rho \alpha^{2} z^{2}, \quad b_{2}=\frac{2 c_{2}}{\alpha^{2} \rho},
\end{aligned}
$$

If $F^{\prime \prime} G^{\prime \prime} \neq 0$. Then, we have to solve (12) to find the functions $F(x)$ and $G(y)$, which is both nonlinear and nonseparable. However, by differentiating first w.r.to $x$ and then w.r.to $y$ this equation becomes

$$
F^{(4)}(x) G^{\prime \prime}(y)-F^{\prime \prime}(x) G^{(4)}(y)=0,
$$

which is in the separable form. Therefore, separating variables, (22) yields the following two linear ordinary differential equations with constant coefficients:

$$
F^{(4)}(x)-\lambda F^{\prime \prime}(x)=0, \quad G^{(4)}(y)-\lambda G^{\prime \prime}(y)=0,
$$

where $\lambda$ is an arbitrary constant.

(a) If $\lambda<0$, let $\lambda=-\beta^{2}, \beta$ being constant, then the solutions of (23) are given by

$$
\begin{gathered}
F(x)=c_{1}+c_{2} x+c_{3} \cos \beta x+c_{4} \sin \beta x, \\
G(y)=d_{1}+d_{2} y+d_{3} \cos \beta y+d_{4} \sin \beta y,
\end{gathered}
$$

where $c_{1}, c_{2}, c_{3}, c_{4}, d_{1}, d_{2}, d_{3}$, and $d_{4}$ are arbitrary constants. Therefore, the stream function $\psi$ is

$$
\begin{aligned}
\psi= & c+c_{2} x+c_{3} \cos \beta x+c_{4} \sin \beta x \\
& +d_{2} y+d_{3} \cos \beta y+d_{4} \sin \beta y
\end{aligned}
$$

where $c=c_{1}+d_{1}$, so that the fluid velocity components are

$$
\begin{gathered}
u=\alpha x+d_{2}-\beta\left(d_{3} \sin \beta y-d_{4} \cos \beta y\right), \\
v=\alpha y-c_{2}+\beta\left(c_{3} \sin \beta x-c_{4} \cos \beta x\right), \\
w=-2 \alpha z .
\end{gathered}
$$

(b) If $\lambda=0$, then solution to (23) is given by

$$
\begin{gathered}
F(x)=c_{1} x^{3}+c_{2} x^{2}+c_{3} x+c_{4}, \\
G(y)=d_{1} y^{3}+d_{2} y^{2}+d_{3} y+d_{4},
\end{gathered}
$$

where $c_{1}, c_{2}, c_{3}, c_{4}, d_{1}, d_{2}, d_{3}$, and $d_{4}$ are arbitrary constants. Thus, the stream function $\psi$ is

$$
\begin{aligned}
\psi(x, y)= & c_{1} x^{3}+d_{1} y^{3}+c_{2} x^{2}+d_{2} y^{2} \\
& +c_{3} x+d_{3} y+d, \quad d=c_{4}+d_{4} .
\end{aligned}
$$

Correspondingly, the fluid velocity components $u, v$, and $w$ along $x, y$, and $z$ axes are given by

$$
\begin{gathered}
u=\alpha x+3 d_{1} y^{2}+2 d_{2} y+d_{3}, \\
v=\alpha y-3 c_{1} x^{2}-2 c_{2} x-c_{3}, \\
w=-2 \alpha z .
\end{gathered}
$$

where $a_{2}$ and $c_{2}$ are arbitrary constants. 
(c) If $\lambda>0$, let $\lambda=\beta^{2}, \beta$ being constant then solution to (23) is given by

$$
\begin{gathered}
F(x)=c_{1}+c_{2} x+c_{3} e^{-\beta x}+c_{4} e^{\beta x}, \\
G(y)=d_{1}+d_{2} y+d_{3} e^{-\beta y}+d_{4} e^{\beta y},
\end{gathered}
$$

where $c_{1}, c_{2}, c_{3}, c_{4}, d_{1}, d_{2}, d_{3}$, and $d_{4}$ are arbitrary constants. Therefore, the stream function $\psi$ is given by

$$
\begin{aligned}
\psi= & d+c_{2} x+c_{3} e^{-\beta x}+c_{4} e^{\beta x} \\
& +d_{2} y+d_{3} e^{-\beta y}+d_{4} e^{\beta y} .
\end{aligned}
$$

Consequently, the velocity components of the fluid are the following:

$$
\begin{gathered}
u=\alpha x+d_{2}-\beta\left(d_{3} e^{-\beta y}-d_{4} e^{\beta y}\right), \\
v=\alpha y-c_{2}+\beta\left(c_{3} e^{-\beta x}-c_{4} e^{\beta x}\right), \\
w=-2 \alpha z .
\end{gathered}
$$

The pressure distribution in all the three cases (a), (b), and (c) is given by

$$
\begin{aligned}
p= & A+B \eta x-\rho \alpha(B y+C) x y \\
& -\frac{\alpha^{2} \rho}{2}\left(x^{2}+y^{2}\right)+\rho(B y+C) F(x) \\
& -\lambda \rho[\alpha x y-F(x)] G(y) \\
& +[\lambda \eta-\rho \alpha] x G^{\prime}(y)-2 \rho \alpha^{2} z^{2} .
\end{aligned}
$$

We remark that all the solutions given in (26), (29), and (32) are free from viscosity coefficient $\eta$ and are known as universal solutions. However, pressure field in each case involves the viscosity coefficient $\eta$. Further note that the solutions are essentially the same as that of the Berker [2] in 2-dimensional case.

4.1. Riabouchinsky Type Flow. Here, we assume that the stream function $\psi$ is linear in $x$ and has the following general form:

$$
\psi(x, y)=x F(y)+G(y),
$$

where $F(y)$ and $G(y)$ are arbitrary functions of $y$. Using (34), Aristov and Gitman [8] obtained an analytical solution of the compatibility equation by taking $F(y)$ as a linear function of $y$. Siddiqui et al. $[9,10]$ studied the same in the context of second grade fluid. Here, we consider some more particular cases of $F(y)$.

On substituting from (34) the compatibility equation (9) yields the following two differential equations:

$$
\begin{gathered}
F \frac{d^{3} F}{d y^{3}}-\frac{d F}{d y} \frac{d^{2} F}{d y^{2}}=\alpha\left[3 \frac{d^{2} F}{d y^{2}}+y \frac{d^{3} F}{d y^{3}}\right]-\nu \frac{d^{4} F}{d y^{4}} \\
F \frac{d^{3} G}{d y^{3}}-\frac{d G}{d y} \frac{d^{2} F}{d y^{2}}=\alpha\left[2 \frac{d^{2} G}{d y^{2}}+y \frac{d^{3} G}{d y^{3}}\right]-\nu \frac{d^{4} G}{d y^{4}}
\end{gathered}
$$

and (35) admits the following particular solutions:

$$
\begin{gathered}
F(y)=a y+b, \\
F(y)=6 \nu\left(y+a_{1}\right)^{-1}-\alpha a_{1}, \\
F(y)=a_{2} e^{-\lambda y}+\alpha\left(y-\frac{4}{\lambda}\right)+\lambda \nu,
\end{gathered}
$$

where $a, a_{1}, a_{2}, b$, and $\lambda$ are arbitrary constants. If $G(y)=$ 0 , then, (37)-(39) completely determine the solution for the stream function $\psi$ and hence for the fluid velocity components $u$ and $v$. Thus, we assume $G(y) \neq 0$ and try to solve (36) in combination with (37)-(39).

(a) On substituting value of the function $F(y)$ from (37) in (36), we obtain

$$
\nu \frac{d^{4} G}{d y^{4}}+[(a-\alpha) y+b] \frac{d^{3} G}{d y^{3}}-2 \alpha \frac{d^{2} G}{d y^{2}}=0 .
$$

Now, setting $U=d^{2} G / d y^{2}$ and choosing the constant $a=\alpha$, (40) reduces to the following equation with constant coefficients:

$$
\begin{gathered}
\nu \frac{d^{2} U}{d y^{2}}+b \frac{d U}{d y}-2 \alpha U=0, \\
\Longrightarrow U(y)=c_{1} e^{m_{1} y}+c_{2} e^{m_{2} y},
\end{gathered}
$$

where $m_{1}=\left(-b+\sqrt{b^{2}+8 \alpha \nu}\right) / 2 v, m_{2}=\left(-b-\sqrt{b^{2}+8 \alpha \nu}\right) / 2 v$ and $c_{1}, c_{2}$ are arbitrary constants. Thus,

$$
G(y)=\frac{c_{1}}{m_{1}^{2}} \cdot e^{m_{1} y}+\frac{c_{2}}{m_{2}^{2}} \cdot e^{m_{2} y}+A y+B
$$

where $A, B$ are arbitrary constants. Therefore, the stream function and the corresponding fluid velocity components are

$$
\begin{gathered}
\psi(x, y)=x(\alpha y+b)+\frac{c_{1}}{m_{1}^{2}} \cdot e^{m_{1} y}+\frac{c_{2}}{m_{2}^{2}} \cdot e^{m_{2} y}+A y+B, \\
u=2 \alpha x+\frac{c_{1}}{m_{1}} e^{m_{1} y}+\frac{c_{2}}{m_{2}} e^{m_{2} y}+A, \\
v=-b, \quad w=-2 \alpha z .
\end{gathered}
$$

(b) Integrating (36) once, letting $U=d G / d y$ in the resulting equation and substituting the value of $F(y)$ from (38), we obtain

$$
\begin{gathered}
v \frac{d^{2} U}{d y^{2}}+\left[6 \nu\left(y+a_{1}\right)^{-1}-\alpha\left(y+a_{1}\right)\right] \frac{d U}{d y} \\
+\left[6 v\left(y+a_{1}\right)^{-2}-\alpha\right] U=0,
\end{gathered}
$$

which is a second order differential equation with variable coefficients. To solve, we assume

$$
U(y)=\sum_{n=0}^{\infty} c_{n}\left(y+a_{1}\right)^{n+r},
$$


where $r$ is a constant to be determined. We obtain the following solution of (45)

$$
\begin{aligned}
U(y)= & C_{1}\left(y+a_{1}\right)^{-3}\left[1-\frac{\alpha\left(y+a_{1}\right)^{2}}{v}\right] \\
& +C_{2}\left(y+a_{1}\right)^{-2}\left[1-\frac{\alpha\left(y+a_{1}\right)^{2}}{3 ! v}-U_{1}(y)\right], \\
U_{1}(y)= & \sum_{n=1}^{\infty}\left(\frac{[1 \cdot 3 \cdot 5 \cdots(2 n-1)] \cdot\left[\alpha\left(y+a_{1}\right)^{2}\right]^{n+1}}{(2 n+3) ! \nu^{n+1}}\right) .
\end{aligned}
$$

Therefore, the function $G(y)$ finally becomes

$$
\begin{aligned}
& G(y)=-C_{1}\left[\frac{\alpha}{v} \ln \left(y+a_{1}\right)+\frac{1}{2}\left(y+a_{1}\right)^{-2}\right] \\
&-C_{2}\left(y+a_{1}\right)^{-1}\left[1+\frac{\alpha y\left(y+a_{1}\right)}{3 ! v}+G_{1}\right], \\
& G_{1}(y)=\sum_{n=1}^{\infty}\left[\frac{1 \cdot 3 \cdot 5 \cdots(2 n-1)}{(2 n+1)(2 n+3) !}\left(\frac{\alpha\left(y+a_{1}\right)^{2}}{v}\right)^{n+1}\right] .
\end{aligned}
$$

Hence, the stream function $\psi$ and the fluid velocity components are given by

$$
\begin{gathered}
\psi(x, y)=\left[6 \nu\left(y+a_{1}\right)^{-1}-\alpha a_{1}\right] x+G(y), \\
u=\alpha x-6 \nu x\left(y+a_{1}\right)^{-2} \\
+C_{1}\left(y+a_{1}\right)^{-3}\left[1-\frac{\alpha\left(y+a_{1}\right)^{2}}{v}\right]+C_{2} u_{1}, \\
v=\alpha\left(y+a_{1}\right)-6 \nu\left(y+a_{1}\right)^{-1}, \\
w=-2 \alpha z, \\
u_{1}=\left(y+a_{1}\right)^{-2}\left[1-\frac{1}{3 !} \cdot \frac{\alpha\left(y+a_{1}\right)^{2}}{v}-U_{1}\right] .
\end{gathered}
$$

It is remarked that by choosing the constant $C_{2}=0$ we may get rid of the power series part and may obtain closed form solution for the stream function $\psi$ and for the fluid velocity component $u$.

(c) On substituting value of the function $F(y)$ from (39) into (36) yields

$$
\begin{aligned}
\nu \frac{d^{4} G}{d y^{4}} & +\left[a_{2} e^{-\lambda y}-\frac{4 \alpha}{\lambda}+\lambda \nu\right] \frac{d^{3} G}{d y^{3}} \\
& -2 \alpha \frac{d^{2} G}{d y^{2}}-a_{2} \lambda^{2} e^{-\lambda y} \frac{d G}{d y}=0 .
\end{aligned}
$$

It is not easy to obtain the general solution of (50). Therefore, we consider a special case when $a_{2}=0$ and obtain

$$
\nu \frac{d^{4} G}{d y^{4}}+\left(\lambda \nu-\frac{4 \alpha}{\lambda}\right) \frac{d^{3} G}{d y^{3}}-2 \alpha \frac{d^{2} G}{d y^{2}}=0 .
$$

In order to solve this equation we reduce its order by letting $U=d^{2} G / d y^{2}$. Then (51) becomes

$$
\nu \frac{d^{2} U}{d y^{2}}+\gamma \frac{d U}{d y}-2 \alpha U=0, \quad \gamma=\lambda \nu-\frac{4 \alpha}{\lambda} .
$$

We notice that for $a_{2}=0$, the particular solution (39) falls back to the solution given in (37) with $a=\alpha$ and $b=\lambda \nu-$ $4 \alpha / \lambda$. This is why (52) is similar to (41) having solution similar to $(42)$, that is,

$$
U(y)=c_{1} e^{m_{1} y}+c_{2} e^{m_{2} y}, \quad m_{1,2}=\frac{-\gamma \pm \sqrt{\gamma^{2}+8 v \alpha}}{2 \nu}
$$

where $c_{1}$ and $c_{2}$ are arbitrary constants. Thus,

$$
G(y)=\frac{c_{1}}{m_{1}^{2}} \cdot e^{m_{1} y}+\frac{c_{2}}{m_{2}^{2}} \cdot e^{m_{2} y}+A y+B,
$$

where $A$ and $B$ are two arbitrary constants. The stream function $\psi$ and the fluid velocity components are

$$
\begin{aligned}
\psi(x, y)= & x\left[\alpha\left(y-\frac{4}{\lambda}\right)+\lambda \nu\right] \\
& +\frac{c_{1}}{m_{1}^{2}} \cdot e^{m_{1} y}+\frac{c_{2}}{m_{2}^{2}} \cdot e^{m_{2} y}+A y+B, \\
u= & 2 \alpha x+\frac{c_{1}}{m_{1}} e^{m_{1} y}+\frac{c_{2}}{m_{2}} e^{m_{2} y}+A, \\
v & =\frac{4 \alpha}{\lambda}-\lambda \nu, \quad w=-2 \alpha z .
\end{aligned}
$$

The analytical expression for the fluid pressure distribution in all the three cases (a), (b), and (c) is given by

$$
\begin{aligned}
p= & \frac{\rho}{2}\left[\left(A x^{2}+B x+C\right)-\alpha^{2}\left(x^{2}+y^{2}\right)\right. \\
& \left.+(2 \alpha y-F) F-2 \nu F^{\prime}\right]-2 \rho \alpha^{2} z^{2} .
\end{aligned}
$$

Remark. If we set $b=0$ in (37), we can recover the case $F(y)=a y$ studied by Aristov and Gitman [8]. Similarly, letting $\alpha=0$ in the cases (38) and (39) additionally studied in this paper, Riabouchinsky type 2-dimensional solutions can be obtained, see Berker [2], Polyanin and Zaitsev [11]. Moreover, the case (39) has also been considered by Siddiqui et al. $[9,10]$ in the context of second grade fluid. However, the special cases considered for evaluation of $G(y)$ they choose $a_{2}=0$ and/or $\alpha=0$. Clearly, choosing $\alpha=0$ means that the plates are at not moving.

4.2. Potential Flow Perturbations. In this section, we look for the fluid motion resulting from the superposition of a potential flow and a perturbation, see Berker [2]. Therefore, the stream function of this type of fluid motion will be of the form

$$
\psi(x, y)=\phi(x, y)+\zeta(x, y)
$$


where the function $\zeta(x, y)$ denotes perturbation and the function $\phi(x, y)$ corresponds to the potential flow of the fluid that satisfies the following equation:

$$
\nabla^{2} \phi=0 .
$$

Now on substituting from (57) and (58) into the compatibility equation (9), we obtain

$$
\begin{aligned}
\nu \nabla^{4} \zeta & -\alpha\left[2 \nabla^{2} \zeta+x \frac{\partial}{\partial x}\left(\nabla^{2} \zeta\right)+y \frac{\partial}{\partial y}\left(\nabla^{2} \zeta\right)\right] \\
& =-\frac{\partial\left(\phi, \nabla^{2} \zeta\right)}{\partial(x, y)}-\frac{\partial\left(\zeta, \nabla^{2} \zeta\right)}{\partial(x, y)} .
\end{aligned}
$$

Let us now suppose that the perturbation $\zeta$ is being restricted to satisfy the following condition:

$$
\nabla^{2} \zeta=f(\zeta)
$$

that is, the vorticity of the perturbation is constant along each streamline of this same perturbation. Therefore, by virtue of (60), the second term on right hand side of (59) disappears and we obtain

$$
\nu \nabla^{4} \zeta-\alpha\left[2 \nabla^{2} \zeta+x \frac{\partial}{\partial x}\left(\nabla^{2} \zeta\right)+y \frac{\partial}{\partial y}\left(\nabla^{2} \zeta\right)\right]=-\frac{\partial\left(\phi, \nabla^{2} \zeta\right)}{\partial(x, y)}
$$

We now consider the special case where the potential function $\phi$ is given by $\phi=a x, a$ being an arbitrary constant, then, the perturbation function $\zeta$ must satisfy the following two equations:

$$
\begin{gathered}
\nabla^{2} \zeta=f(\zeta) \\
\nu \nabla^{4} \zeta-\alpha\left[2 \nabla^{2} \zeta+x \frac{\partial}{\partial x}\left(\nabla^{2} \zeta\right)+y \frac{\partial}{\partial y}\left(\nabla^{2} \zeta\right)\right]=-\frac{\partial}{\partial y}\left(\nabla^{2} \zeta\right) .
\end{gathered}
$$

To find a particular solution of this system, we assume that $f(\zeta)=\zeta$ and $\zeta$ is a homogeneous function of degree $n$ of the variables $x$ and $y$. Therefore, the solution of the system (62) and (63) is given by

$$
\zeta(x, y)=\Theta(x) e^{k y},
$$

where $k=(n+2 \alpha-\nu) / a$ is constant and $\Theta$ is an arbitrary function of $x$. The stream function $\psi$ is given by

$$
\begin{aligned}
& \psi(x, y)=a x+\left(c_{1} e^{\sqrt{1-k^{2}} x}+c_{2} e^{-\sqrt{1-k^{2}} x}\right) e^{k y} \quad \text { if } k^{2}<1, \\
& \psi(x, y)=a x+\left(c_{1}+c_{2} x\right) e^{k y} \quad \text { if } k^{2}=1, \\
& \psi(x, y)=a x+\left(c_{1} \cos \left(k^{2}-1\right) x+c_{2} \sin \left(k^{2}-1\right) x\right) e^{k y}, \\
& \text { if } k^{2}>1 \text {. }
\end{aligned}
$$

Knowing stream function, the velocity components of the fluid motion and the expressions for pressure distribution can be easily found.

\section{References}

[1] P. F. Neményi, "Recent developments in inverse and semiinverse methods in the mechanics of continua," Advances in Applied Mechanics, vol. 2, pp. 123-151, 1951.

[2] R. Berker, "Integration des equations du mouvement d'un fluide visqueux incompressible," in Handbuch der Physik, S. Flugge, Ed., vol. 2, pp. 1-384, Springer, Berlin, Germany, 1963.

[3] H. L. Dryden, F. D. Murnaghan, and F. D. Bateman, "Report of the committee on hydrodynamics," Bulletin National Research Council, vol. 84, pp. 155-332, 1932.

[4] G. B. Whitham, “The Navier-Stokes equations of motion”, in Laminar Boundary Layers, L. Rosenhead, Ed., pp. 114-162, Clarendon Press, Oxford, UK, 1963.

[5] H. Schlichting, Boundary-Layer Theory, McGraw-Hill, New York, NY, USA, 6th edition, 1968.

[6] C. Y. Wang, "Fluid flow due to a stretching cylinder," Physics of Fluids, vol. 31, no. 3, pp. 466-468, 1988.

[7] C. Y. Wang, "Exact solutions of the steady-state Navier-Stokes equations," Annual Review of Fluid Mechanics, vol. 23, no. 1, pp. 159-177, 1991.

[8] S. N. Aristov and I. M. Gitman, "Viscous flow between two moving parallel disks: exact solutions and stability analysis," Journal of Fluid Mechanics, vol. 464, pp. 209-215, 2002.

[9] A. M. Siddiqui, M. R. Mohyuddin, T. Hayat, and S. Asghar, "Some more inverse solutions for steady flows of a second-grade fluid," Archives of Mechanics, vol. 55, no. 4, pp. 373-387, 2003.

[10] A. M. Siddiqui, "Some more inverse solutions of a nonnewtonian fluid," Mechanics Research Communications, vol. 17, no. 3, pp. 157-163, 1990.

[11] A. D. Polyanin and V. F. Zaitsev, Handbook of Nonlinear Partial Differential Equations, Chapman \& Hall/CRC, Boca Raton, Fla, USA, 2003. 


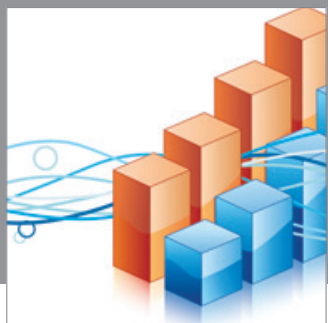

Advances in

Operations Research

mansans

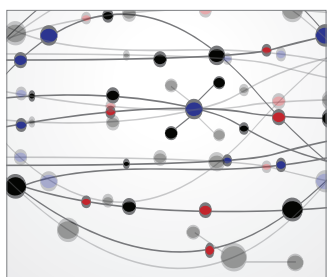

The Scientific World Journal
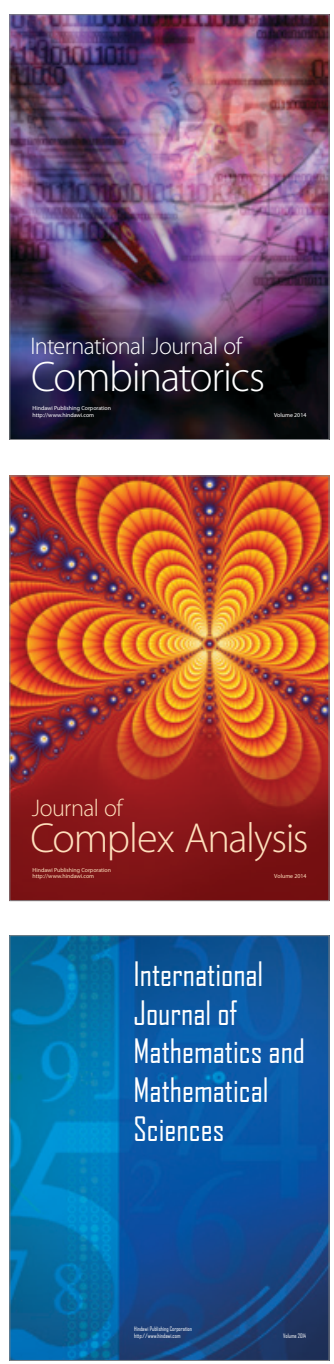
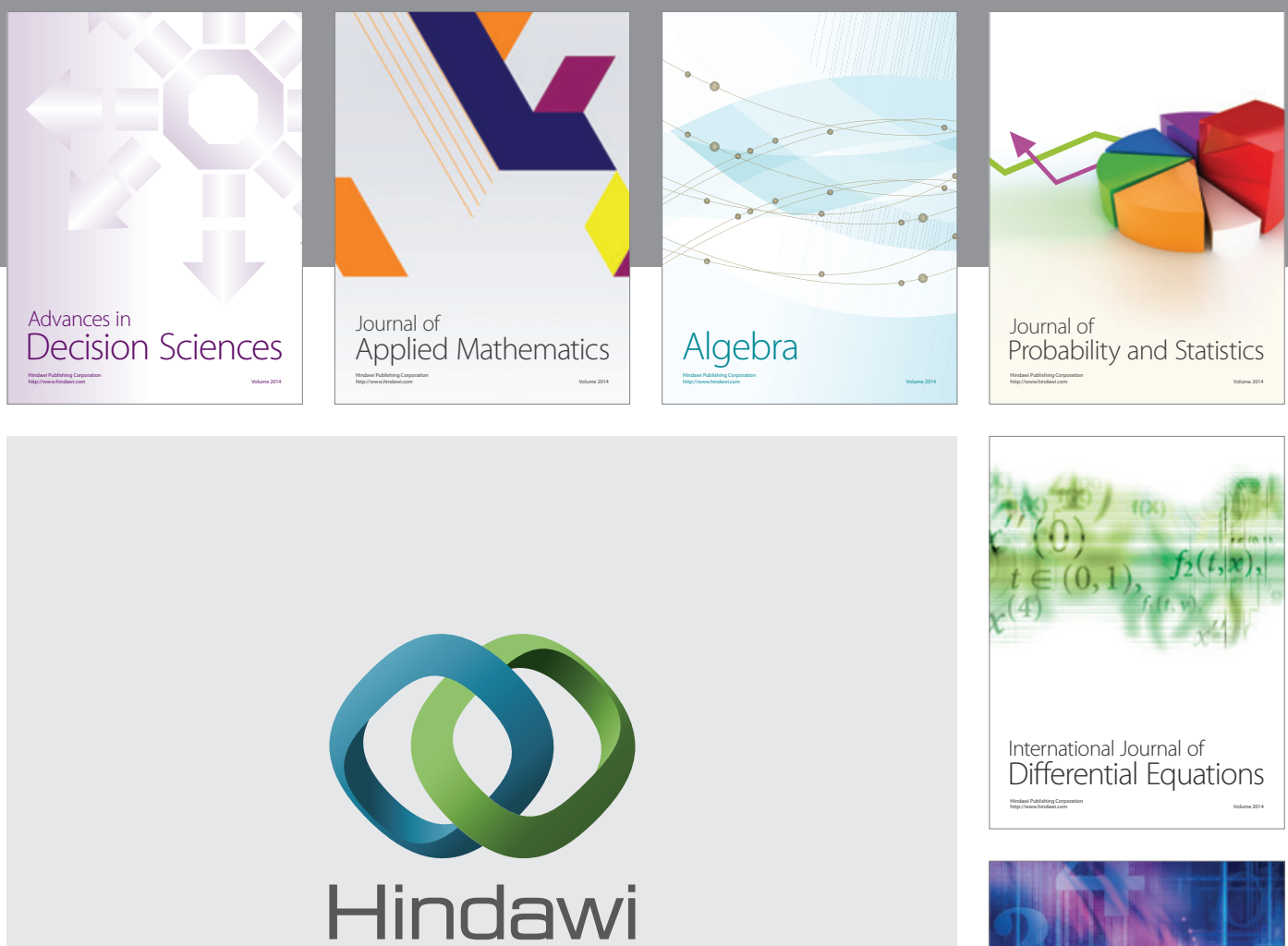

Submit your manuscripts at http://www.hindawi.com
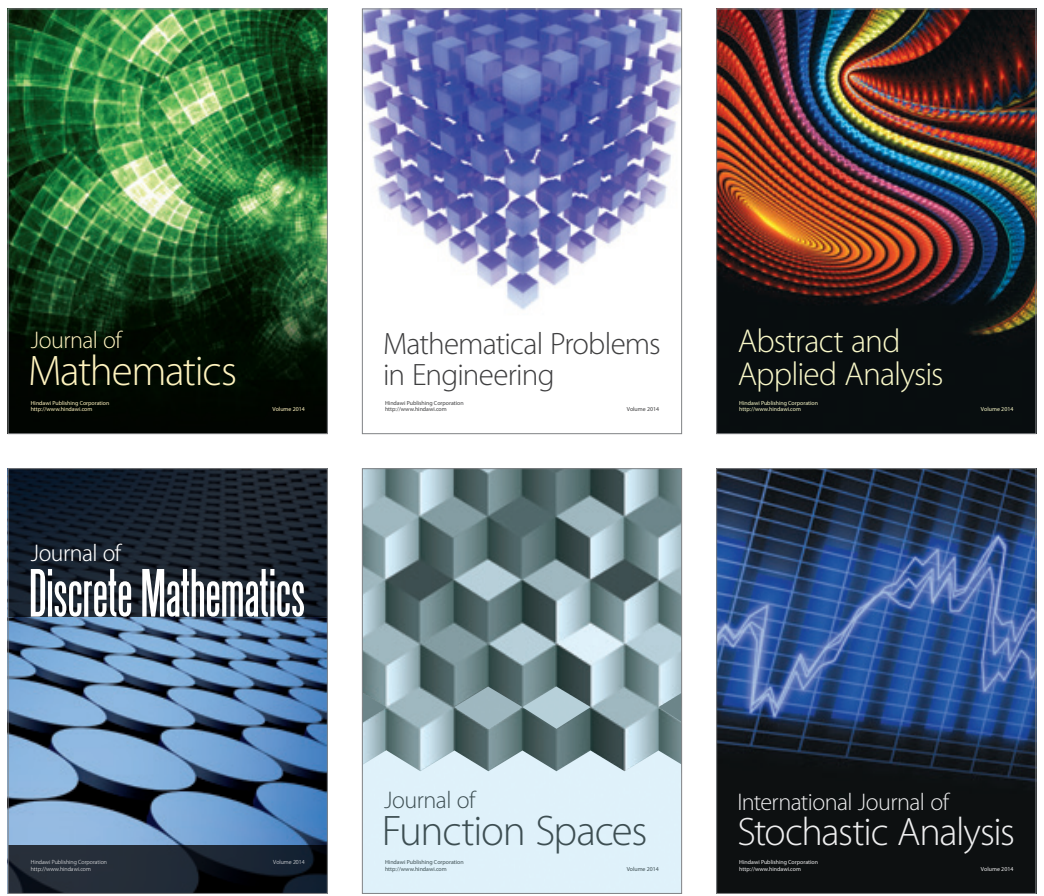

Journal of

Function Spaces

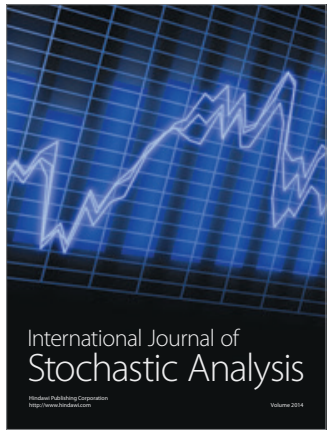

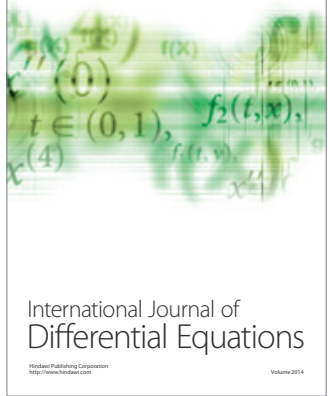
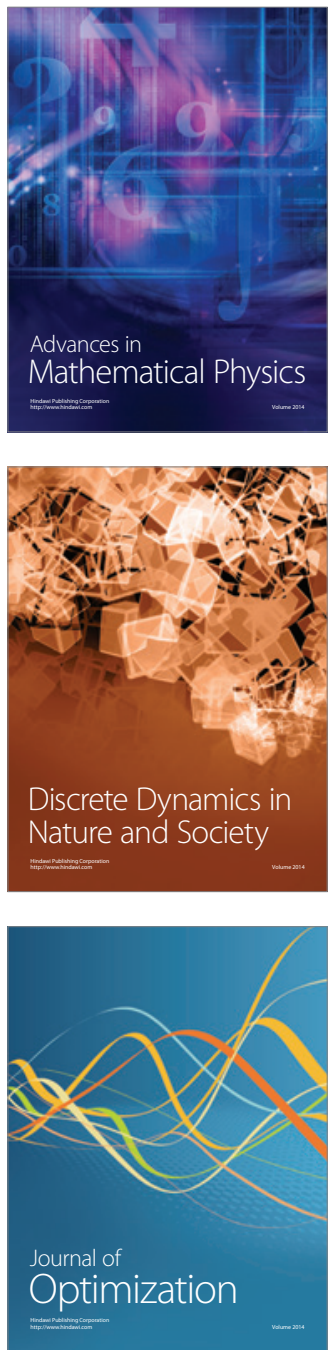